\title{
Quasi-static deformation due to two-dimensional seismic sources embedded in an elastic half-space in welded contact with a poroelastic half-space
}

\author{
Sunita Rani ${ }^{1}$ and Sarva Jit Singh ${ }^{2}$ \\ ${ }^{1}$ Department of Mathematics, Guru Jambheshwar University, Hisar 125 001, India. \\ e-mail: s_b_rani@rediffmail.com \\ ${ }^{2}$ INSA Senior Scientist, Department of Mathematics, University of Delhi, South Campus, \\ New Delhi 110 021, India. \\ e-mail: s_j_singh@yahoo.com
}

The Biot linearized theory of fluid saturated porous materials is used to study the plane strain deformation of a two-phase medium consisting of a homogeneous, isotropic, poroelastic half-space in welded contact with a homogeneous, isotropic, perfectly elastic half-space caused by a twodimensional source in the elastic half-space. The integral expressions for the displacements and stresses in the two half-spaces in welded contact are obtained from the corresponding expressions for an unbounded elastic medium by applying suitable boundary conditions at the interface. The case of a long dip-slip fault is discussed in detail. The integrals for this source are solved analytically for two limiting cases: (i) undrained conditions in the high frequency limit, and (ii) steady state drained conditions as the frequency approaches zero. It has been verified that the solution for the drained case $(\omega \rightarrow 0)$ coincides with the known elastic solution. The drained and undrained displacements and stresses are compared graphically. Diffusion of the pore pressure with time is also studied.

\section{Introduction}

The theory of poroelasticity studies the timedependent coupling between the deformation of rock and fluid flow within the rock. The study of deformation of a fluid-saturated porous medium by buried sources is important for its geophysical applications. Biot (1941, 1956) developed linearized constitutive and field equations for poroelastic media which have been used very extensively (see, e.g., Wang (2000) and the references listed therein).

Rongved (1955) derived closed-form algebraic expressions for the Papkowich-Neuber displacement potentials for an arbitrary point force acting in an infinite medium consisting of two elastic half-spaces in welded contact. Using the Rongved solution, Heaton and Heaton (1989) obtained the deformation field induced by point forces and point force couples embedded in two Poissonian halfspaces in welded contact. Kumari et al (1992) generalized the results of Heaton and Heaton (1989) by obtaining analytical expressions for the deformation field valid for arbitrary Poisson's ratio. The corresponding two-dimensional problem has been discussed by Singh et al (1992).

The problem of a point source in a poroelastic medium has been discussed by several researchers (see, e.g., Chau 1996; Pan 1999; Rice and Clearly 1976; Rudnicki 1986). The corresponding two-dimensional case has been considered, among others, by Rudnicki (1987), Rudnicki and Roeloffs (1990) and Singh and Rani (2006).

Keywords. Dip-slip fault; plane strain; poroelastic; seismic source; welded half-spaces. 
In the present paper, we study the plane strain deformation of a two-phase medium consisting of a homogeneous, isotropic, poroelastic half-space in welded contact with a homogeneous, isotropic, elastic half-space due to two-dimensional seismic sources embedded in the elastic half-space. We begin with the integral expression for the Airy stress function for a line source in an unbounded elastic medium given by Singh and Garg (1986) and obtain the integral expressions for stresses and displacements in the two media, using suitable boundary conditions at the interface. The integrals cannot be evaluated analytically for arbitrary values of the frequency. Two particular cases are studied in detail for a dip-slip line dislocation: Case I, when the frequency tends to infinity corresponding to the undrained condition, and Case II, when the frequency approaches zero corresponding to the drained conditions. As expected, the results for the drained state coincide with the corresponding results for a source in two elastic half-spaces in welded contact given by Singh et al (1992).

\section{Theory}

We consider a two-dimensional approximation in which the displacement components $\left(u_{1}, u_{2}, u_{3}\right)$ are independent of the Cartesian coordinate $x_{2}$ so that $\partial / \partial x_{2} \equiv 0$. Under this assumption, the plane strain problem $\left(u_{2}=0\right)$ and the antiplane strain problem $\left(u_{1}=u_{3}=0\right)$ get decoupled, and can therefore be treated independently. Since the antiplane deformation is not affected by pore pressure, we shall confine our discussion to plane strain problem only.

Let the Cartesian coordinates be denoted by $(x, y, z) \equiv\left(x_{1}, x_{2}, x_{3}\right)$ with $z$-axis vertically

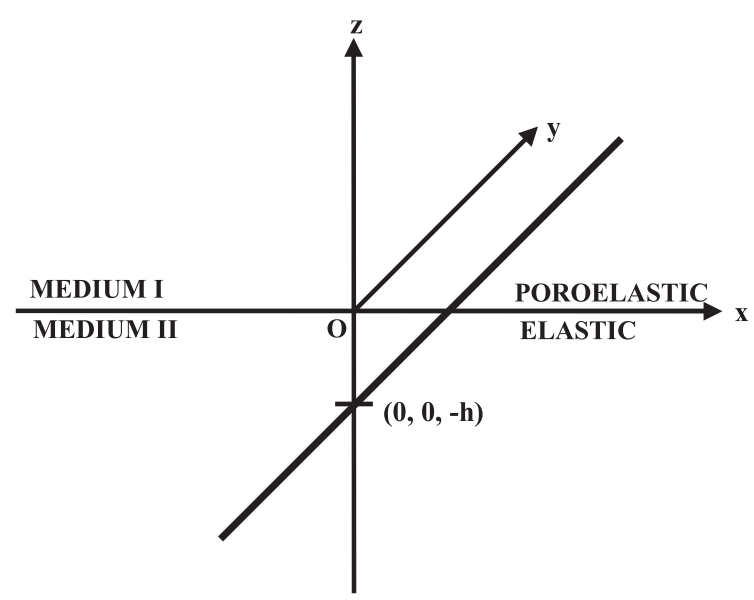

Figure 1. A line source parallel to the $y$ axis located at the point $(0,0,-h)$ of an elastic half-space $(z<0$, Medium II) in welded contact with a poroelastic half-space $(z>0$, Medium I). upwards. Consider two homogeneous isotropic half-spaces which are welded along the plane $z=0$ (figure 1 ). The upper half-space $(z>0)$ is poroelastic and is called medium I. The lower half-space $(z<0)$ is perfectly elastic and is called medium II.

\subsection{Solution for poroelastic half-space}

A homogeneous, isotropic, poroelastic medium can be characterized by five poroelastic parameters: shear modulus $(G)$, drained Poisson's ratio $(\nu)$, undrained Poisson's ratio $\left(\nu_{u}\right)$, Skempton's coefficient $(B)$ and hydraulic diffusivity $(c)$. Darcy conductivity $(\chi)$ and Biot-Willis coefficient $(\alpha)$ can be expressed in terms of these five parameters:

$$
\begin{gathered}
\chi=\frac{9 c\left(1-\nu_{u}\right)\left(\nu_{u}-\nu\right)}{2 G B^{2}(1-\nu)\left(1+\nu_{u}\right)^{2}}, \\
\alpha=\frac{3\left(\nu_{u}-\nu\right)}{B(1-2 \nu)\left(1+\nu_{u}\right)} .
\end{gathered}
$$

The plane strain problem for an isotropric poroelastic medium can be solved in terms of Biot's stress function $F$ such that (Biot 1956; Singh and Rani 2006)

$$
\begin{aligned}
& \sigma_{11}=\frac{\partial^{2} F}{\partial z^{2}}, \quad \sigma_{33}=\frac{\partial^{2} F}{\partial x^{2}}, \quad \sigma_{13}=-\frac{\partial^{2} F}{\partial x \partial z}, \\
& \nabla^{2}\left(\nabla^{2} F+2 \eta p\right)=0, \\
& \left(c \nabla^{2}-\frac{\partial}{\partial t}\right)\left[\nabla^{2} F+\frac{3}{\left(1+\nu_{u}\right) B} p\right]=0,
\end{aligned}
$$

where $\sigma_{i j}$ denotes the total stress in the fluidsaturated porous elastic material, $p$ the excess fluid pore pressure (compression negative) and

$$
\eta=\frac{1-2 \nu}{2(1-\nu)} \alpha
$$

is the poroelastic stress coefficient.

Eliminating $F$ and $p$ in turn, equations (4) and (5) lead us to the following decoupled equations

$$
\begin{aligned}
& \left(c \nabla^{2}-\frac{\partial}{\partial t}\right) \nabla^{2} p=0, \\
& \left(c \nabla^{2}-\frac{\partial}{\partial t}\right) \nabla^{4} F=0 .
\end{aligned}
$$

The general solution of equation (7) may be expressed in the form

$$
p=p_{1}+p_{2},
$$


where

$$
\begin{gathered}
c \nabla^{2} p_{1}=\frac{\partial p_{1}}{\partial t}, \\
\nabla^{2} p_{2}=0 .
\end{gathered}
$$

Similarly, the general solution of equation (8) may be expressed in the form

$$
F=F_{1}+F_{2},
$$

where

$$
\begin{aligned}
c \nabla^{2} F_{1} & =\frac{\partial F_{1}}{\partial t}, \\
\nabla^{4} F_{2} & =0 .
\end{aligned}
$$

Separation of time and space variables can be made for each of the four functions: $p_{1}, p_{2}, F_{1}$ and $F_{2}$. Assuming the time-dependence in the form $\exp (-\iota \omega t)$, equations (10), (11), (13) and (14) yield

$$
\begin{aligned}
& \nabla^{2} p_{1}+\frac{\iota \omega}{c} p_{1}=0, \\
& \nabla^{2} p_{2}=0, \\
& \nabla^{2} F_{1}+\frac{\iota \omega}{c} F_{1}=0, \\
& \nabla^{4} F_{2}=0,
\end{aligned}
$$

where $p_{1}, p_{2}, F_{1}$ and $F_{2}$ are now functions of $x$ and $z$ only.

Suitable solutions of equations (15)-(18) can be found by the method of separation of variables. Using equations (9) and (12), we may take

$$
\begin{gathered}
p=\int_{0}^{\infty}\left(A_{1} e^{-m z}+A_{2} e^{-k z}\right)\left(\begin{array}{c}
\sin k x \\
\cos k x
\end{array}\right) d k, \\
F=\int_{0}^{\infty}\left[B_{1} e^{-m z}+\left(B_{2}+B_{3} k z\right) e^{-k z}\right]\left(\begin{array}{c}
\sin k x \\
\cos k x
\end{array}\right) d k,
\end{gathered}
$$

where

$$
m=\left(\frac{c k^{2}-\iota \omega}{c}\right)^{1 / 2} \quad(\operatorname{Re} m>0),
$$

and $A_{i}, B_{i}$ may be functions of $k$. From equations (4), (5), (19) and (20), we find

$$
\begin{aligned}
& A_{1}=\frac{\iota \omega}{2 \eta c} B_{1}, \\
& A_{2}=\frac{2}{3}\left(1+\nu_{u}\right) B k^{2} B_{3} .
\end{aligned}
$$

From equations (3) and (20), the stresses are found to be

$$
\begin{aligned}
\sigma_{11}= & \int_{0}^{\infty}\left[B_{1} m^{2} e^{-m z}+\left(B_{2}-2 B_{3}+B_{3} k z\right) k^{2} e^{-k z}\right] \\
& \times\left(\begin{array}{c}
\sin k x \\
\cos k x
\end{array}\right) d k, \\
\sigma_{33}= & -\int_{0}^{\infty}\left[B_{1} e^{-m z}+\left(B_{2}+B_{3} k z\right) e^{-k z}\right] \\
& \times\left(\begin{array}{c}
\sin k x \\
\cos k x
\end{array}\right) k^{2} d k, \\
\sigma_{13}= & \int_{0}^{\infty}\left[\begin{array}{c}
{\left[B_{1} m e^{-m z}+\left(B_{2}-B_{3}+B_{3} k z\right) k e^{-k z}\right]} \\
\end{array}\right. \\
& \times\left(\begin{array}{c}
\cos k x \\
-\sin k x
\end{array}\right) k d k .
\end{aligned}
$$

Corresponding to the stresses (24)-(26), the displacements are given by (Singh and Rani 2006)

$$
\begin{aligned}
2 G u_{1}= & -\int_{0}^{\infty}\left[B_{1} e^{-m z}\right. \\
& \left.+\left\{B_{2}+B_{3}\left(2 \nu_{u}-2+k z\right)\right\} e^{-k z}\right] \\
& \times\left(\begin{array}{c}
\cos k x \\
-\sin k x
\end{array}\right) k d k, \\
2 G u_{3}= & \int_{0}^{\infty}\left[B_{1} m e^{-m z}\right. \\
& \left.+\left\{B_{2}+B_{3}\left(1-2 \nu_{u}+k z\right)\right\} k e^{-k z}\right] \\
& \times\left(\begin{array}{c}
\sin k x \\
\cos k x
\end{array}\right) d k,
\end{aligned}
$$

where

$$
\alpha_{0}=\frac{3\left(\nu_{u}-\nu\right)}{B\left(1+\nu_{u}\right)}
$$

Darcy's flux in the $z$-direction $(q)$ is given by

$$
\begin{aligned}
q & =-\chi \frac{\partial p}{\partial z} \\
& =\chi \int_{0}^{\infty}\left(m A_{1} e^{-m z}+k A_{2} e^{-k z}\right)\left(\begin{array}{c}
\sin k x \\
\cos k x
\end{array}\right) d k,
\end{aligned}
$$

where equation (19) has been used. 
Table 1. Source coefficients for various sources $\left[\alpha^{\prime}=1 /\left\{2\left(1-\nu^{\prime}\right)\right\}\right]$.

\begin{tabular}{|c|c|c|c|}
\hline Source & $S_{1}$ & $S_{2}$ & $\begin{array}{l}\text { Upper or lower } \\
\text { solution }\end{array}$ \\
\hline Single couple (23) & $-\in \frac{F_{23}}{2 \pi}$ & $\in \alpha^{\prime} \frac{F_{23}}{2 \pi}$ & Upper \\
\hline Single couple (32) & $\in \frac{F_{32}}{2 \pi}$ & $\in \alpha^{\prime} \frac{F_{32}}{2 \pi}$ & Upper \\
\hline $\begin{array}{l}\text { Double couple }(23)+(32) \\
\left(F_{23}=F_{32}=D_{23}\right)\end{array}$ & 0 & $\in \alpha^{\prime} \frac{D_{23}}{\pi}$ & Upper \\
\hline $\begin{array}{l}\text { Centre of rotation }(32)-(23) \\
\left(F_{23}=F_{32}=R_{23}\right)\end{array}$ & $\frac{\in R_{23}}{\pi}$ & 0 & Upper \\
\hline Dipole (22) & $\left(1-\alpha^{\prime}\right) \frac{F_{22}}{2 \pi}$ & $-\alpha^{\prime} \frac{F_{22}}{2 \pi}$ & Lower \\
\hline Dipole (33) & $\left(1-\alpha^{\prime}\right) \frac{F_{33}}{2 \pi}$ & $\alpha^{\prime} \frac{F_{33}}{2 \pi}$ & Lower \\
\hline $\begin{array}{l}\text { Centre of dilatation }(22)+(33) \\
\left(F_{22}=F_{33}=C_{0}\right)\end{array}$ & $\left(1-\alpha^{\prime}\right) \frac{C_{0}}{\pi}$ & 0 & Lower \\
\hline $\begin{array}{l}\text { Double couple }(33)-(22) \\
\left(F_{22}=F_{33}=D_{23}^{\prime}\right)\end{array}$ & 0 & $\alpha^{\prime} \frac{D_{23}^{\prime}}{\pi}$ & Lower \\
\hline Tensile dislocation in $x_{2}$-direction & $\alpha^{\prime} \frac{T_{0}}{\pi}$ & $-\alpha^{\prime} \frac{T_{0}}{\pi}$ & Lower \\
\hline Tensile dislocation in $x_{3}$-direction & $\alpha^{\prime} \frac{T_{0}}{\pi}$ & $\alpha^{\prime} \frac{T_{0}}{\pi}$ & Lower \\
\hline
\end{tabular}

\subsection{Solution for elastic half-space}

A homogeneous isotropic elastic medium can be characterized by two elastic parameters: shear modules $\left(G^{\prime}\right)$ and Poisson's ratio $\left(\nu^{\prime}\right)$. The plane strain problem for an isotropic elastic medium can be solved in terms of the Airy stress function $\Phi$ such that

$$
\sigma_{11}^{\prime}=\frac{\partial^{2} \Phi}{\partial z^{2}}, \quad \sigma_{33}^{\prime}=\frac{\partial^{2} \Phi}{\partial x^{2}}, \quad \sigma_{13}^{\prime}=-\frac{\partial^{2} \Phi}{\partial x \partial z},
$$

$$
\nabla^{2} \nabla^{2} \Phi=0
$$

where $\sigma_{i j}^{\prime}$ are the stress components.

Let there be a line source parallel to the $y$-axis passing through the point $(0,0,-h)$ of the elastic half space $(z<0$, figure 1$)$. As shown by Singh and Garg (1986), the Airy stress function for a line source parallel to the $y$ axis passing through the point $(0,0,-h)$ in an unbounded isotropic elastic medium can be expressed in the form

$$
\Phi_{0}=\int_{0}^{\infty}\left(S_{1}+S_{2} \in k Z\right) e^{-\in k Z}\left(\begin{array}{c}
\sin k x \\
\cos k x
\end{array}\right) \frac{d k}{k},
$$

where

$$
Z=z+h, \quad \in= \pm 1,
$$

the upper sign is for $Z>0$ and the lower sign for $Z<0$. The source coefficients $S_{1}$ and $S_{2}$ are independent of $k$. Singh and Garg (1986) have obtained these coefficients for various seismic sources. These are listed in table 1 for ready reference. The upper or lower solution in the last column of table 1 refers to equation (32). We use the notation of Ben-Menahem and Singh (1981) for labelling various sources. Thus, (23) denotes the single couple in the $x_{2} x_{3}$ plane with forces in the $x_{2}$-direction and $F_{23}$ is the corresponding moment; $(23)+(32)$ denotes the double couple in the $x_{2} x_{3}$-plane; (32)-(23) denotes the centre of rotation in the $x_{2} x_{3}$-plane; (22) denotes the dipole in the $x_{2}$-direction of strength $F_{22} ;(22)+(33)$ denotes the centre of dilatation and (33)-(22) denotes the double couple whose forces bisect the angles between the dipoles (22) and (33). In table 1, we have included the source coefficients for a tensile dislocation as well for which $T_{0}=G^{\prime} b d s$, where $b$ is the displacement discontinuity and $d s$ is the width of the dislocation.

For a line source parallel to the $y$-axis (figure 1 ) acting at the point $(0,0,-h)$ of the elastic halfspace $(z<0)$ which is in welded contact with the poroelastic half-space $(z>0)$, the Airy stress function for the elastic half-space may be taken to be of the form

$$
\Phi=\Phi_{0}+\int_{0}^{\infty}\left(C_{1}+C_{2} k z\right) e^{k z}\left(\begin{array}{c}
\sin k x \\
\cos k x
\end{array}\right) d k
$$


where the unknowns $C_{1}, C_{2}$ are to be determined from the boundary conditions and $\Phi_{0}$ is given by equation (32).

From equations (30), (32) and (34), we obtain the following expressions for the stresses in the elastic half-space $(z<0)$ :

$$
\begin{aligned}
\sigma_{11}^{\prime}= & \int_{0}^{\infty}\left[\left\{S_{1}+S_{2}(-2+\in k Z)\right\} e^{-\in k Z}\right. \\
& \left.+\left\{C_{1}+C_{2}(2+k z)\right\} k e^{k z}\right] \\
& \times\left(\begin{array}{c}
\sin k x \\
\cos k x
\end{array}\right) k d k, \\
\sigma_{33}^{\prime}= & -\int_{0}^{\infty}\left[\left(S_{1}+S_{2} \in k Z\right) e^{-\in k Z}\right. \\
& \left.+\left(C_{1}+C_{2} k z\right) k e^{k z}\right]\left(\begin{array}{c}
\sin k x \\
\cos k x
\end{array}\right) k d k, \\
\sigma_{13}^{\prime}= & \int_{0}^{\infty}\left[\left\{S_{1}-S_{2}(1-\in k Z)\right\} \in e^{-\in k Z}\right. \\
& \left.-\left\{C_{1}+C_{2}(1+k z)\right\} k e^{k z}\right] \\
& \times\left(\begin{array}{c}
\cos k x \\
-\sin k x
\end{array}\right) k d k .
\end{aligned}
$$

The corresponding displacements are given by

$$
\begin{aligned}
2 G^{\prime} u_{1}^{\prime}= & \int_{0}^{\infty}\left[\left\{-S_{1}+S_{2}\left(2-2 \nu^{\prime}-\in k Z\right)\right\} e^{-\in k Z}\right. \\
& \left.-\left\{C_{1}+C_{2}\left(2-2 \nu^{\prime}+k z\right)\right\} k e^{k z}\right] \\
& \times\left(\begin{array}{c}
\cos k x \\
-\sin k x
\end{array}\right) d k, \\
2 G^{\prime} u_{3}^{\prime}= & \int_{0}^{\infty}\left[\left\{S_{1}+S_{2}\left(1-2 \nu^{\prime}+\in k Z\right)\right\} \in e^{-\in k Z}\right. \\
& \left.+\left\{-C_{1}+C_{2}\left(1-2 \nu^{\prime}-k z\right)\right\} k e^{k z}\right] \\
& \times\left(\begin{array}{c}
\sin k x \\
\cos k x
\end{array}\right) d k .
\end{aligned}
$$

\section{Boundary conditions}

Since the half-spaces are assumed to be in welded contact along the plane $z=0$, the continuity of the stresses and the displacements give the following boundary conditions

$$
\sigma_{13}=\sigma_{13}^{\prime}, \quad \sigma_{33}=\sigma_{33}^{\prime}
$$

$$
u_{1}=u_{1}^{\prime}, \quad u_{3}=u_{3}^{\prime},
$$

at $z=0$. If we assume that the interface is impermeable, the hydraulic boundary condition is

$$
q=0 \quad \text { at } \quad z=0 .
$$

It is noticed from table 1 that the source coefficients $S_{1}$ and $S_{2}$ might have different values for $Z \gtrless 0$. Let $S_{1}^{\prime}$ and $S_{2}^{\prime}$ be the values of $S_{1}$ and $S_{2}$, respectively, valid for $Z>0$. The boundary conditions (40) and (41) yield the following system of equations:

$$
\begin{gathered}
m B_{1}+k B_{2}-k B_{3}+k C_{1}+k C_{2} \\
=\left(S_{1}^{\prime}-S_{2}^{\prime}+S_{2}^{\prime} k h\right) e^{-k h}, \\
k B_{1}+k B_{2}-k C_{1}=\left(S_{1}^{\prime}+S_{2}^{\prime} k h\right) e^{-k h}, \\
k B_{1}+k B_{2}+2 k\left(\nu_{u}-1\right) B_{3}-\theta k C_{1} \\
-2 \theta k\left(1-\nu^{\prime}\right) C_{2} \\
=\theta\left(S_{1}^{\prime}+S_{2}^{\prime} k h-2 S_{2}^{\prime}+2 S_{2}^{\prime} \nu^{\prime}\right) e^{-k h}, \\
m B_{1}+k B_{2}+k\left(1-2 \nu_{u}\right) B_{3}+\theta k C_{1} \\
-\theta k\left(1-2 \nu^{\prime}\right) C_{2} \\
=\theta\left(S_{1}^{\prime}+S_{2}^{\prime}-2 S_{2}^{\prime} \nu^{\prime}+S_{2}^{\prime} k h\right) e^{-k h}, \\
m A_{1}+k A_{2}=0,
\end{gathered}
$$

where

$$
\theta=\frac{G}{G^{\prime}} .
$$

Solving the system of equations (42)-(46) and using equations (22) and (23), we obtain

$$
\begin{aligned}
A_{1}= & \frac{\Omega}{2 \eta} Q(k+m) S_{2}^{\prime} e^{-k h}, \\
A_{2}= & -\frac{m}{k} A_{1}, \\
B_{1}= & \frac{\Omega Q}{k-m} S_{2}^{\prime} e^{-k h}, \\
B_{2}= & {\left[-\frac{P_{2}}{k}\left(S_{1}^{\prime}-\frac{S_{2}^{\prime}}{2}+S_{2}^{\prime} k h\right)\right.} \\
& \left.+\frac{Q S_{2}}{2 k}\left(1-\frac{k+m}{k-m} \Omega\right)\right] e^{-k h},
\end{aligned}
$$




$$
\begin{aligned}
B_{3}= & \frac{Q}{K} S_{2}^{\prime} e^{-k h}, \\
C_{1}= & {\left[-\frac{P_{1}}{k}\left(S_{1}^{\prime}+S_{2}^{\prime} k h\right)\right.} \\
& \left.+\frac{S_{2}^{\prime}}{2 k}\left\{P_{2}+(1+\Omega) Q\right\}\right] e^{-k h}, \\
C_{2}= & \frac{P_{1}}{k}\left[2 S_{1}^{\prime}-S_{2}^{\prime}+2 S_{2}^{\prime} k h\right] e^{-k h},
\end{aligned}
$$

where

$$
\begin{aligned}
P_{1} & =\frac{1-\theta}{1+3 \theta-4 \theta \nu^{\prime}}, \quad P_{2}=P_{1}-1, \\
P_{3} & =\frac{4 \nu_{u}-3-\theta}{1-\theta}, \\
Q & =\frac{P_{2}}{P_{1}\left(P_{3}+\Omega\right)}, \quad \Omega=\frac{k^{2} \gamma}{m(m+k)}, \\
\gamma & =\frac{2\left(\nu-\nu_{u}\right)}{(1-\nu)} .
\end{aligned}
$$

Substituting the values of $A_{1}, B_{1}$, etc. in equations (19), (20) and (34), we get the integral expressions for the stress functions in the two media and pore pressure in terms of the source coefficients $S_{1}$ and $S_{2}$. The values of these coefficients for various sources are given in table 1 . The expressions for a particular source are obtained on putting the values of the source coefficients for that source. However, the integrals cannot be solved analytically for arbitrary values of $\omega$. Two particular cases of great interest are: Case I as the frequency $\omega \rightarrow \infty$ corresponding to the undrained state and Case II as $\omega \rightarrow 0$ corresponding to the drained state (Wang 2000; Section 7.5). For these two cases, the integrals appearing in the expressions for the stress functions in the two media and pore pressure can be evaluated using standard integral transform tables. The displacements and stresses can be obtained similarly. Here, we give the results for a two-dimensional dip-slip dislocation.

\section{Vertical dip-slip dislocation}

As shown by Maruyama (1966), the double coupe $(23)+(32)$ is equivalent to a vertical dip-slip line dislocation such that

$$
D_{23}=G^{\prime} b d s,
$$

where $b$ is the slip and $d s$ is the width of the line dislocation. Therefore, from table 1 ,

$$
S_{1}^{\prime}=0, \quad S_{2}^{\prime}=\frac{\alpha^{\prime}}{\pi} D_{23}=\frac{D_{23}}{2 \pi\left(1-\nu^{\prime}\right)},
$$

with the stipulation that in the representation of equation (32), the upper solution is to be selected.

\subsection{Case I (Undrained state, $\omega \rightarrow \infty$ )}

On taking the limit $\omega \rightarrow \infty$ and evaluating the resulting integrals analytically, we obtain, for the poroelastic half-space $(z>0)$,

$$
\begin{aligned}
F= & \frac{\alpha^{\prime} D_{23}}{\pi}\left[\frac{1}{2}\left(P_{2}+Q_{2}\right) \tan ^{-1}\left(\frac{x}{z+h}\right)\right. \\
& \left.+\left(Q_{2} z-P_{2} h\right) \frac{x}{R_{1}^{2}}\right] \\
\sigma_{11}= & \frac{\alpha^{\prime} D_{23}}{\pi}\left(\frac{x}{R_{1}^{4}}\right) \\
& \times\left[P_{2}(z+3 h)-Q_{2}(5 z+3 h)\right. \\
& \left.+8\left(Q_{2} z-P_{2} h\right)\left(\frac{z+h}{R_{1}}\right)^{2}\right], \\
\sigma_{13}= & \frac{\alpha^{\prime} D_{23}}{\pi}\left(\frac{1}{R_{1}^{2}}\right)\left[\frac{1}{2}\left(Q_{2}-P_{2}\right)\right. \\
& +\left\{P_{2}(z+7 h)-Q_{2}(7 z+h)\right\} \\
& \left.\times \frac{z+h}{R_{1}^{2}}+8\left(Q_{2} z-P_{2} h\right) \frac{(z+h)^{3}}{R_{1}^{4}}\right], \\
& +8\left(Q_{2} z-P_{2} h\right)\left(\frac{\alpha^{\prime} D_{23}}{\pi}\left(\frac{x}{R_{1}^{4}}\right)\right. \\
\sigma_{33} & \times\left[P_{2}(z+3 h)-Q_{2}(z-h)\right. \\
& {[5}
\end{aligned}
$$

$$
\begin{aligned}
2 G u_{1}= & \frac{\alpha^{\prime} D_{23}}{\pi}\left(\frac{1}{R_{1}^{2}}\right) \\
& \times\left[-\frac{1}{2}\left\{P_{2}(z+3 h)+Q_{2}(h-z)\right.\right. \\
& \left.\left.-4 Q_{4}(z+h)\right\}-2\left(Q_{2} z-P_{2} h\right) \frac{(z+h)^{2}}{R_{1}^{2}}\right],
\end{aligned}
$$




$$
\begin{aligned}
2 G u_{3}= & \frac{\alpha^{\prime} D_{23}}{\pi}\left(\frac{x}{R_{1}^{2}}\right) \\
& \times\left[\frac{1}{2}\left(P_{2}-Q_{2}+4 Q_{4}\right)\right. \\
& \left.+2\left(Q_{2} z-P_{2} h\right) \frac{z+h}{R_{1}^{2}}\right], \\
p= & -\frac{\alpha^{\prime} D_{23}}{\pi}\left(\frac{\gamma}{\eta}\right) Q_{2} x(z+h) \frac{1}{R_{1}^{4}},
\end{aligned}
$$

where

$$
\begin{gathered}
Q_{2}=\frac{P_{2}}{P_{1} P_{3}}, \quad Q_{4}=\left(1-\nu_{u}\right) Q_{2}, \\
R_{1}^{2}=x^{2}+(z+h)^{2}, \quad R_{2}^{2}=x^{2}+(z-h)^{2} .
\end{gathered}
$$

Similarly, for the elastic half-space $(z<0)$, we find

$$
\begin{aligned}
\Phi= & \frac{\alpha^{\prime} D_{23}}{\pi}\left[\frac{1}{2}\left(P_{2}+Q_{2}\right) \tan ^{-1}\left(\frac{x}{h-z}\right)\right. \\
& +x(z+h) \frac{1}{R_{1}^{2}}-P_{1} x(z+h) \frac{1}{R_{2}^{2}} \\
& \left.+4 P_{1} x h z(h-z) \frac{1}{R_{2}^{4}}\right] \\
\sigma_{11}^{\prime}= & \frac{\alpha^{\prime} D_{23}}{\pi} x\left[2\left\{-3+4\left(\frac{z+h}{R_{1}}\right)^{2}\right\} \frac{z+h}{R_{1}^{4}}\right. \\
& +\left\{\left(P_{2}+Q_{2}\right)(h-z)-2 P_{1}(5 h-3 z)\right. \\
& +8 P_{1}(h-z)\left(z^{2}+3 h^{2}-10 h z\right) \frac{1}{R_{2}^{2}} \\
& \left.\left.+96 P_{1} h z \frac{(h-z)^{3}}{R_{2}^{4}}\right\} \frac{1}{R_{2}^{4}}\right], \\
\sigma_{13}^{\prime}= & \frac{\alpha^{\prime} D_{23}}{\pi}\left[\left\{1-8\left(\frac{z+h}{R_{1}}\right)^{2}+8\left(\frac{z+h}{R_{1}}\right)^{4}\right\} \frac{1}{R_{1}^{2}}\right. \\
& +\left\{\frac{1}{2}\left(P_{2}-2 P_{1}+Q_{2}\right)+\left(8 P_{1}-P_{2}-Q_{2}\right)\right. \\
& (61)
\end{aligned}
$$

$$
\begin{aligned}
2 G^{\prime} u_{3}^{\prime}= & \frac{\alpha^{\prime} D_{23}}{\pi} x\left[\left\{1-2 \nu^{\prime}+2\left(\frac{z+h}{R_{1}}\right)^{2}\right\} \frac{1}{R_{1}^{2}}\right. \\
& -\left\{\frac{1}{2}\left(P_{2}+Q_{2}\right)+P_{1}\left(1-2 \nu^{\prime}\right)\right\} \frac{1}{R_{2}^{2}} \\
& +2 P_{1}\left\{3 h^{2}-z^{2}-4 \nu^{\prime} h(h-z)\right\} \frac{1}{R_{2}^{4}} \\
& \left.-16 P_{1} h z(h-z)^{2} \frac{1}{R_{2}^{6}}\right]
\end{aligned}
$$

4.2 Case II (Drained state, $\omega \rightarrow 0$ )

The results for the limiting case $\omega \rightarrow 0$ can be obtained similarly. We define 


$$
\begin{aligned}
P_{4} & =\frac{4 \nu-3-\theta}{1-\theta}, \quad Q_{1}=\frac{P_{2}}{P_{1} P_{4}}, \\
Q_{3} & =(1-\nu) Q_{1}
\end{aligned}
$$

and note that

$$
\frac{P_{3}+\frac{1}{2} \gamma}{1+\frac{1}{2} \gamma}=P_{4}, \quad \frac{P_{3}+\frac{1}{2} \gamma}{1-\nu_{u}}=\frac{P_{4}}{1-\nu} .
$$

On taking the limit $\omega \rightarrow 0$, evaluating the resulting integrals analytically and using equations (66) and (67), we find that $p=0$ and the expressions for the stresses and the displacements can be obtained from equations (53)-(57) and (61)-(65) on replacing $Q_{2}$ by $Q_{1}$ and $Q_{4}$ by $\mathrm{Q}_{3}$. We have verified that the expressions for the stresses and the displacements valid for $\omega \rightarrow 0$ for source in an elastic half-space in welded contact with a poroelastic
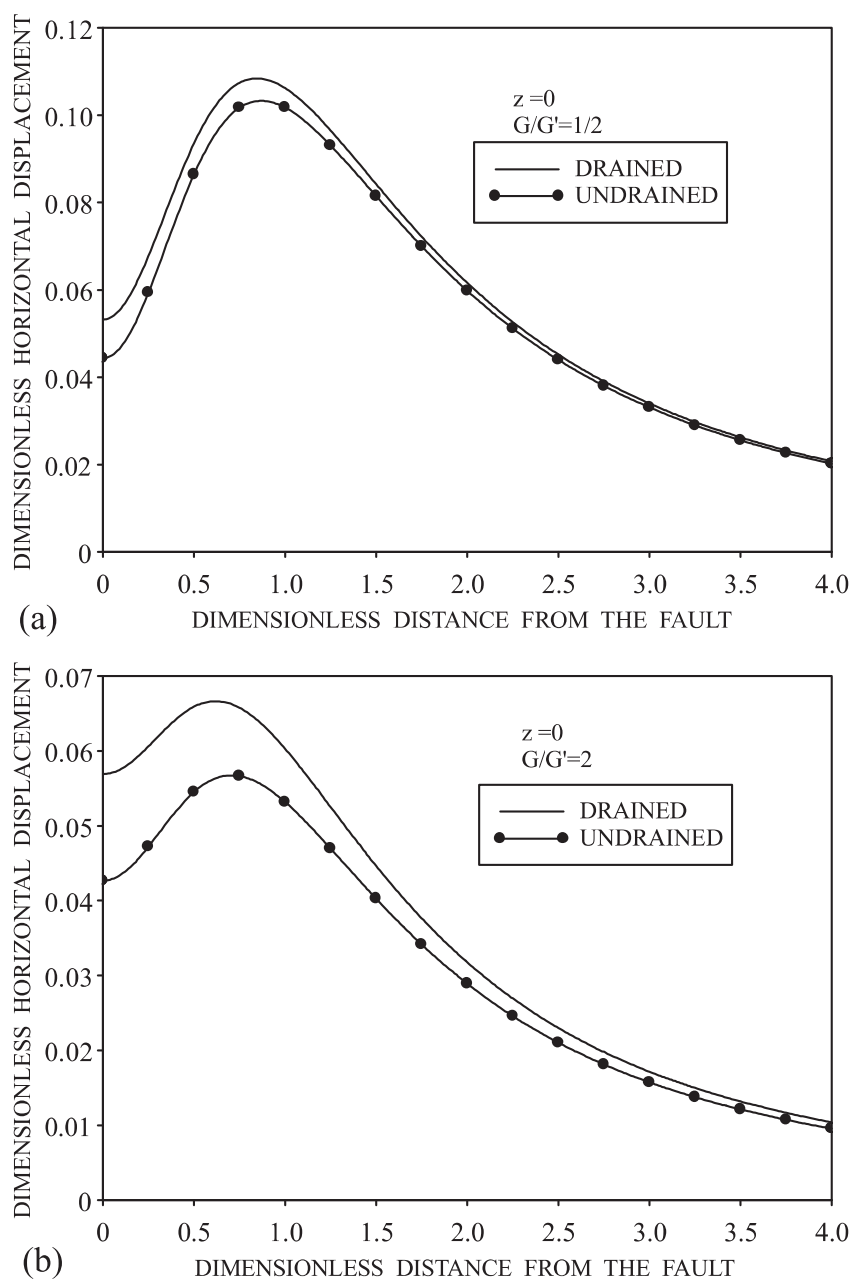

Figure 2. Variation of the horizontal displacement at the interface $(z=0)$ with the horizontal distance from the fault for $\nu=0.12, \nu_{u}=0.31, \nu^{\prime}=0.25$ and for (a) $G / G^{\prime}=0.5$; (b) $G / G^{\prime}=2$. The horizontal displacement is measured in units of $(b d s / h)$ and the horizontal distance is measured in units of $h$. half-space coincide with the corresponding expressions for a source in an elastic half-space in welded contact with another elastic half-space given by Singh et al (1992).

\section{Numerical results and discussion}

\subsection{Drained and undrained states}

Equations (53)-(57) and (61)-(65) can be used for computing the stress and the displacement fields in the two half-spaces under undrained conditions. The pore pressure in the poroelastic half-space is given by equation (58). For the stress and the displacement fields under drained conditions, we have to change $Q_{2}$ to $Q_{1}$ and $Q_{4}$ to $Q_{3}$ in equations (53)-(57) and (61)-(65). We have computed the displacement and stress fields generated by a vertical dip-slip dislocation located at the point $(0,0,-h)$ of the elastic half-space (figure 1$)$. We have assumed that $\nu^{\prime}=0.25, \nu=0.12, \nu_{u}=0.31$. The numerical values of the drained and undrained
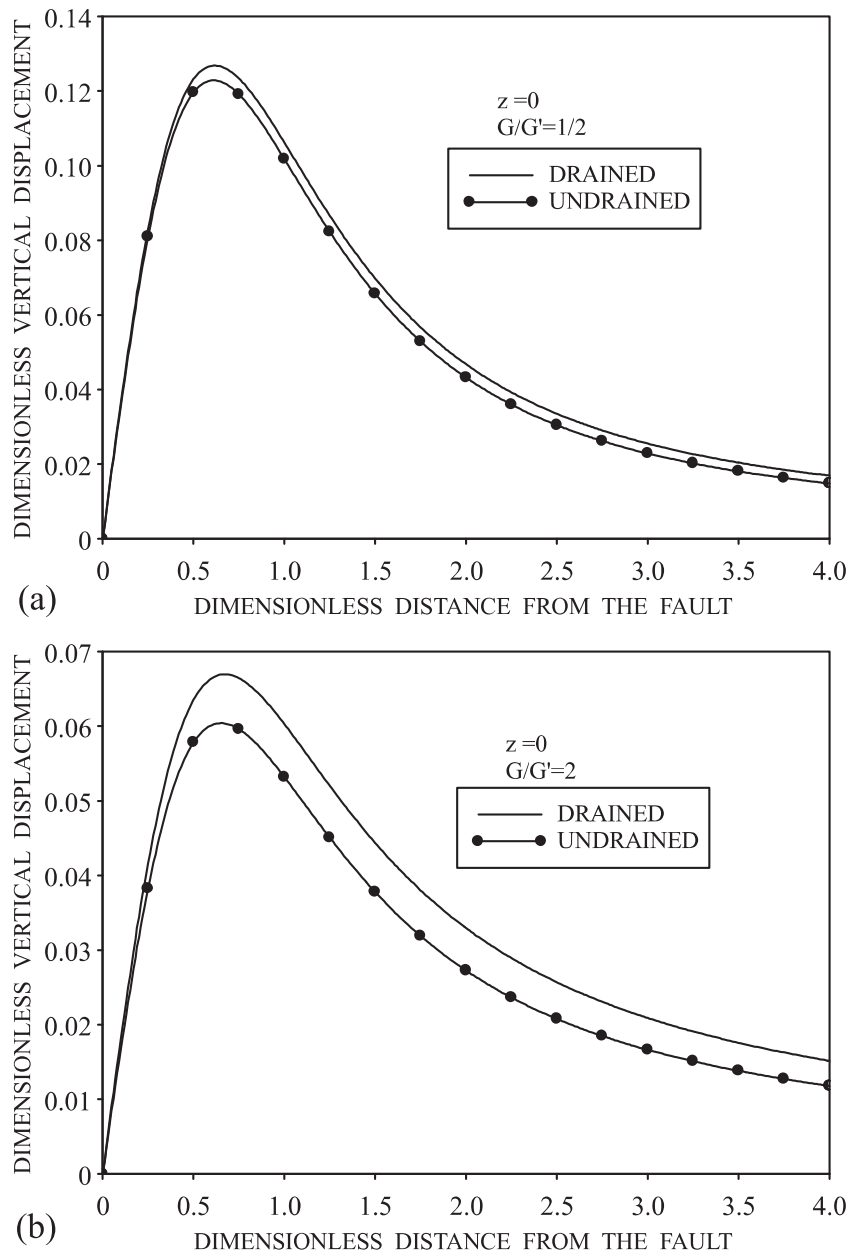

Figure 3. Variation of the vertical displacement for (a) $G / G^{\prime}=0.5$; (b) $G / G^{\prime}=2$. 

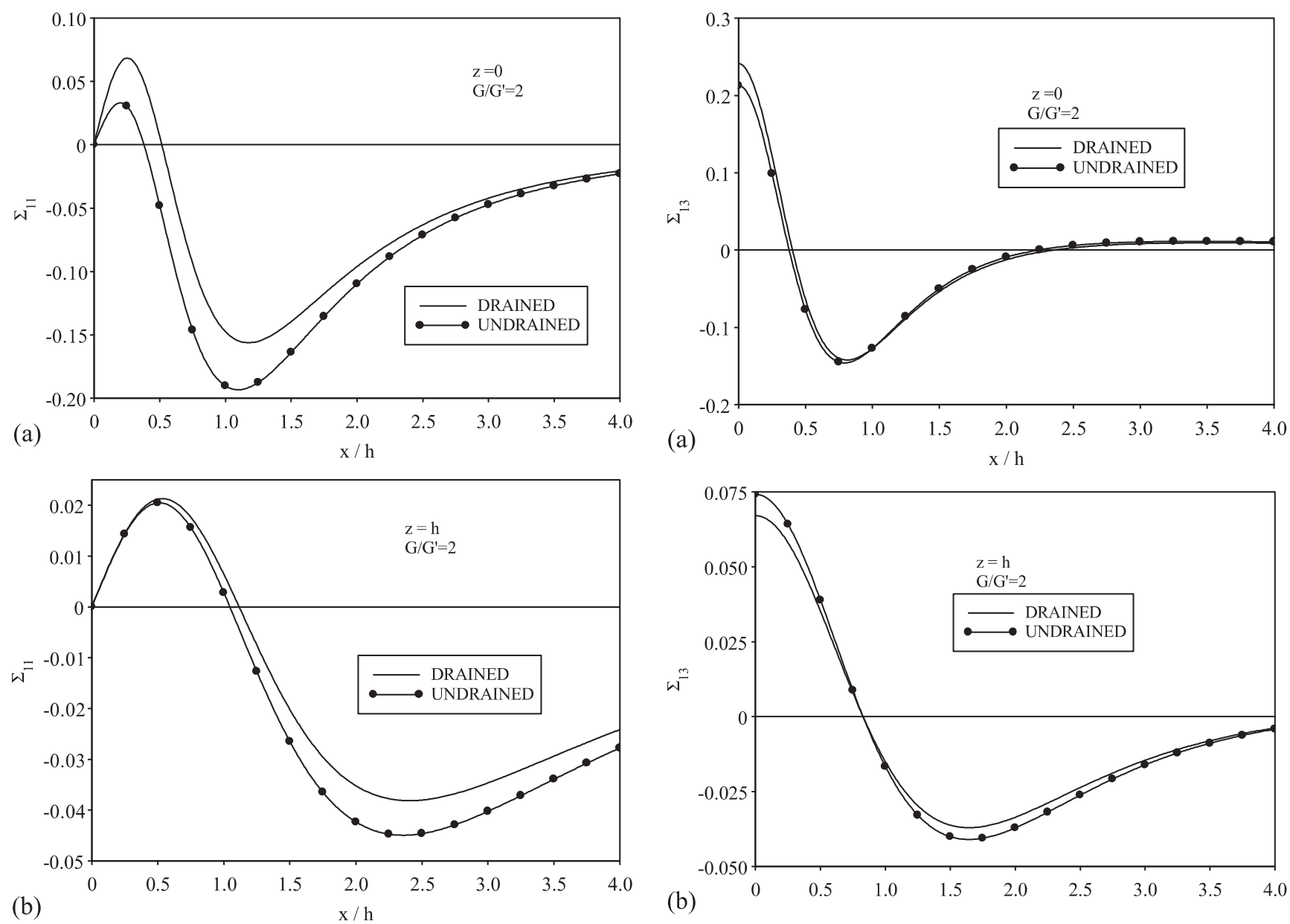

Figure 4. Variation of the dimensionless stress $\Sigma_{11}$ in the poroelastic half-space with the horizontal distance from the fault for $\nu=0.12, \nu_{u}=0.31, \nu^{\prime}=0.25, G / G^{\prime}=2$ and for (a) $z=0$; (b) $z=h$.

Poisson's ratios assumed for the poroelastic halfspace correspond to the Ruhr Sandstone (Wang 2000; p. 266).

Figure 2(a) shows the variation of the dimensionless horizontal displacement with the dimensionless horizontal distance from the fault for $G / G^{\prime}=0.5$. The horizontal displacement $u_{1}$ is measured in units of $(b d s / h)$, where $b$ is the displacement dislocation, $d s$ the width of the fault and $h$ the distance of the line source from the interface. The horizontal distance $x$ is measured in units of $h$. Figure 2(b) is for $G / G^{\prime}=2$. We note that the difference between the drained and the undrained displacements is more when the poroelastic half-space is harder than the elastic half-space as against the case when the poroelastic half-space is softer than the elastic half-space. Figure 3(a) shows the variation of the dimensionless vertical displacement with the dimensionless horizontal distance for $G / G^{\prime}=0.5$. Figure 3(b) is for $G / G^{\prime}=2.0$. Here also, $u_{3}$ is measured in units of $(b d s / h)$ and $x$ in units of $h$.

Figure 5. Variation of the dimensionless stress $\Sigma_{13}$ for (a) $z=0$; (b) $z=h$.

We have given closed-form expressions for pore pressure, stresses and displacements in two limiting cases, viz., $\omega \rightarrow \infty$ (undrained state) and $\omega \rightarrow 0$ (drained state). For arbitrary $\omega$, the integrals occurring in the expressions for pore pressure, stresses and displacements cannot be evaluated analytically. One has to resort to numerical integration.

We define the following dimensionless quantities:

$$
\begin{gathered}
T=\frac{2 c t}{h^{2}}, \\
P=\left(\frac{h^{2}}{G^{\prime} b d s}\right) p, \\
\Sigma_{i j}=\left(\frac{h^{2}}{G^{\prime} b d s}\right) \sigma_{i j},
\end{gathered}
$$

where $h$ is the distance of the line source from the interface, $b$ is the displacement dislocation, $d s$ is the fault width, $G^{\prime}$ is the rigidity of the elastic 

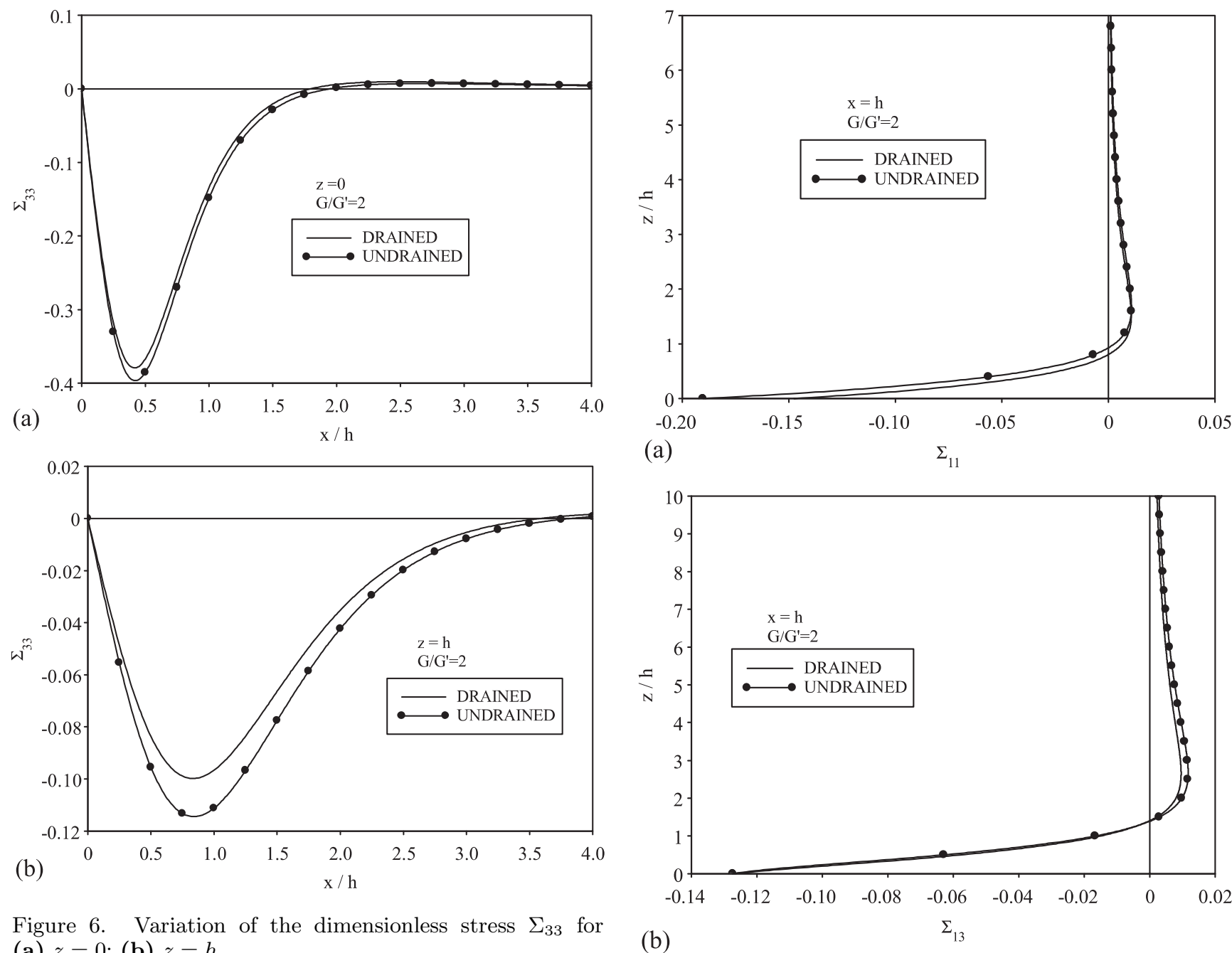

Figure 6. Variation of the dimensionless stress $\Sigma_{33}$ for (a) $z=0$; (b) $z=h$.

half-space and $c$ is the hydraulic diffusivity of the poroelastic half-space.

Figures 4 to 6 show the variation of the dimensionless stresses $\Sigma_{i j}$ with the dimensionless horizontal distance from the fault for $G / G^{\prime}=2$. While figures 4(a), 5(a) and 6(a) are for the stresses at the interface, figures 4(b), 5(b) and 6(b) are for $z=h$ in the poroelastic half-space. The stresses $\sigma_{13}$ and $\sigma_{33}$ are continuous at the interface. But $\sigma_{11}$ is not continuous there. Figure $4\left(\right.$ a) is for $\Sigma_{11}$ in the poroelastic half-space as we approach the interface.

Figure $7(\mathrm{a}, \mathrm{b}, \mathrm{c})$ shows the variation of the dimensionless stresses $\Sigma_{11}, \Sigma_{13}$ and $\Sigma_{33}$ in the poroelastic half-space with dimensionless distance from the interface for $x=h, G / G^{\prime}=2$. The stresses in the poroelastic half-space tend to zero as the distance from the interface tends to infinity.

\subsection{Results in the time domain}

From equations (19), (24) to (27), (35) to (39), (48) and (51), we get the integral expressions for

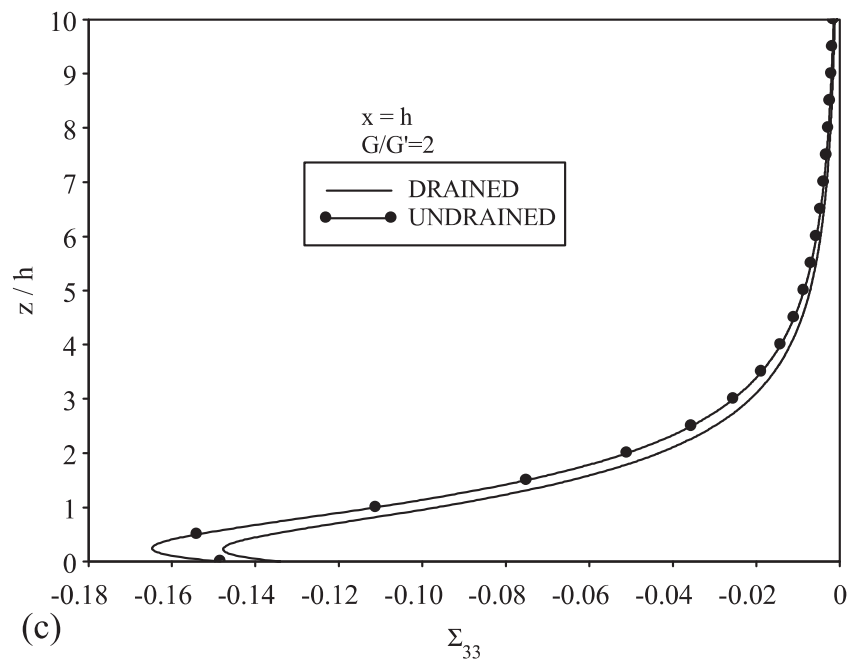

Figure 7. Variation of the dimensionless stress components (a) $\Sigma_{11} ;$ (b) $\Sigma_{13} ;$ (c) $\Sigma_{33}$ in the poroelastic half-space with the distance from the interface for $x=h, \nu=0.12$, $\nu_{u}=0.31, \nu^{\prime}=0.25$ and $G / G^{\prime}=2$.

the pore pressure, displacements and stresses at any point in each of the two half-spaces caused by a vertical dip-slip dislocation located at the 


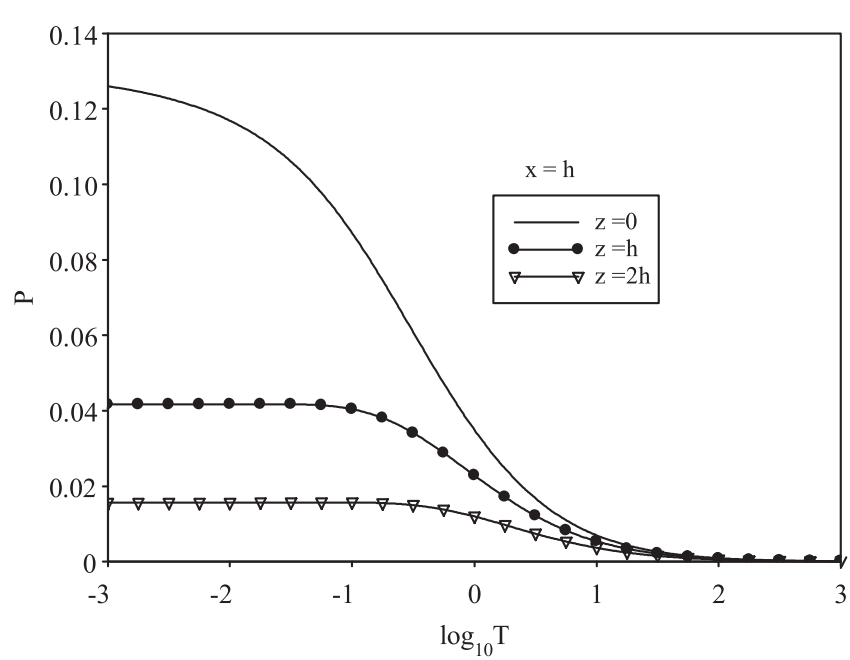

Figure 8. Diffusion of the dimensionless pore pressure $P$ with dimensionless time $T$ for $x=h$ and $z=0, h, 2 h$.
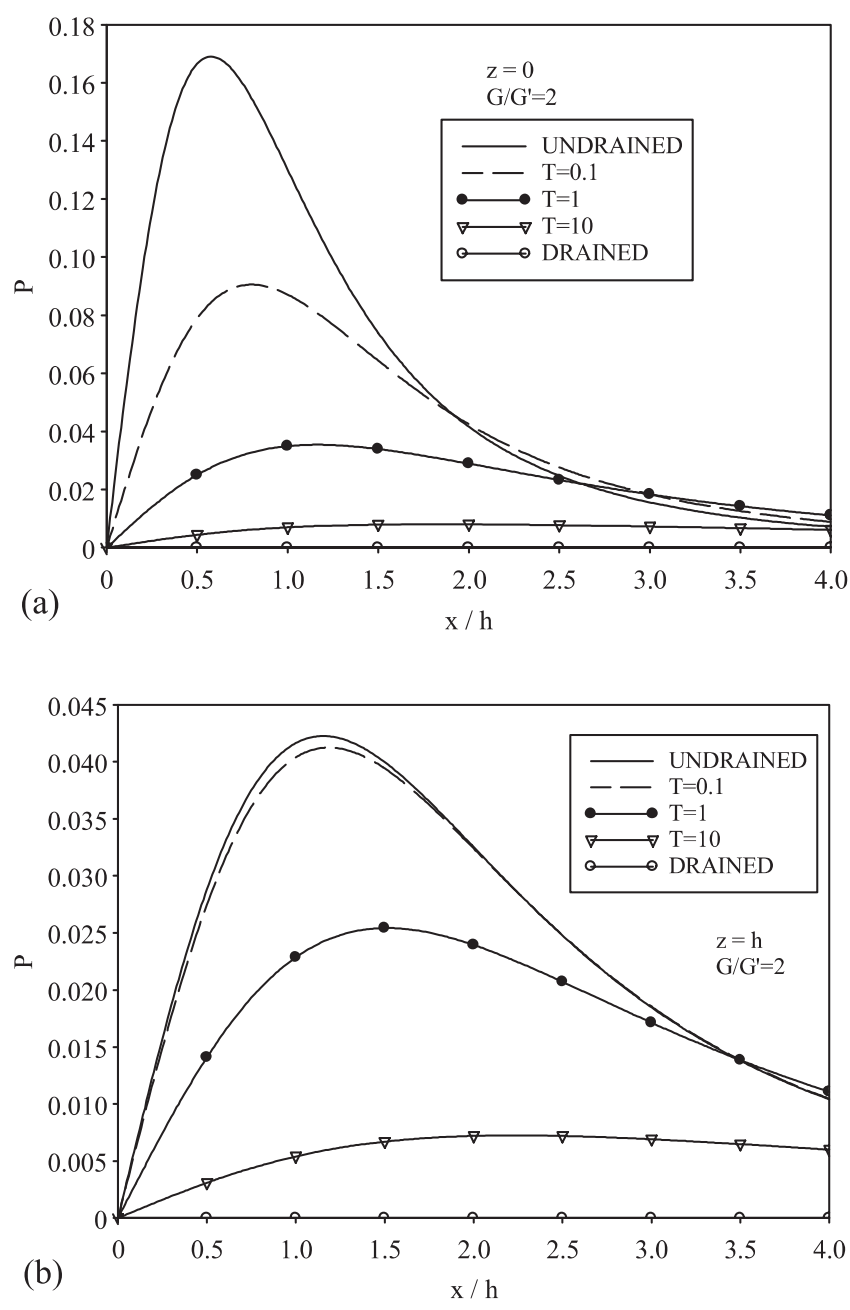

Figure 9. Variation of the dimensionless pore pressure $P$ in the poroelastic half-space with the horizontal distance from the fault at five times: $T=0$ (undrained response), $0.1,1,10, \infty$ (drained response) for (a) $z=0$; (b) $z=h$.

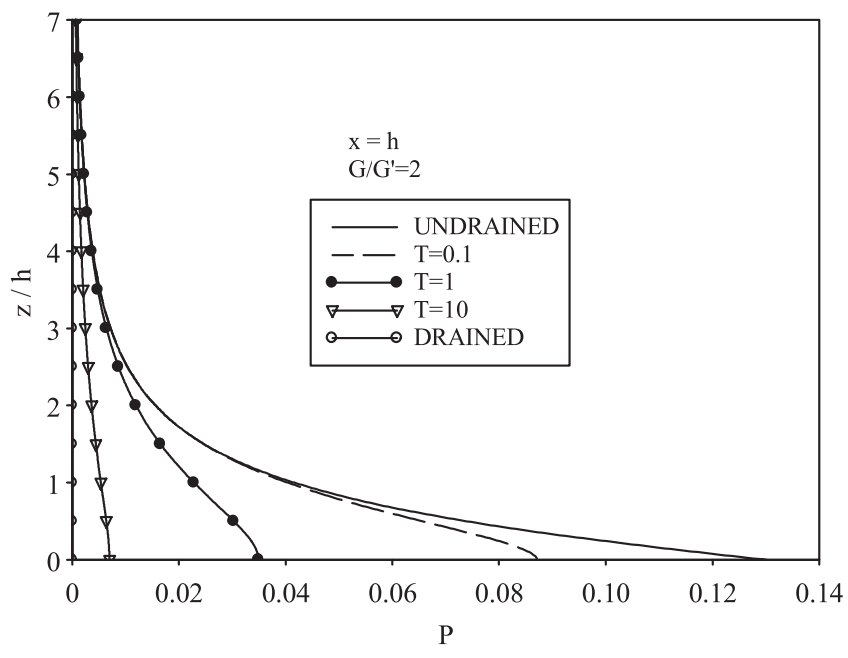

Figure 10. Variation of the dimensionless pore pressure in the poroelastic half-space with the distance from the interface for $x=h$ at five times.

point $(0,0,-h)$ of the elastic half-space. This solution is in the $k-\omega$ plane. On replacing $(-\iota \omega)$ by $s$, we get the solution in the Fourier-Laplace transform domain, where $s$ is the Laplace transform variable. Two integrations are required to be performed to get the solution in the space-time domain.

Schapery (1962) proposed a very simple and efficient approximate formula for finding Laplace inversion numerically. According to this formula

$$
\phi(t) \approx[s \bar{\phi}(s)]_{s=1 /(2 t)},
$$

where $\bar{\phi}(s)$ is the Laplace transform of $\phi(t)$. Accuracy of this formula has been demonstrated, amongst others, by Rajapakse and Senjuntichai (1993), Senjuntichai and Rajapakse (1995) and Chau (1996). We have used Schapery's approximate formula for Laplace inversion.

Fourier transform inversion involves evaluating the semi-infinite integral with respect to $k$. This has been done by using extended Simpson's rule. The semi-infinite integral is discretized into a series of integrals over sub-intervals delimited by consecutive zeros of the integrand and truncated after achieving the desired accuracy. Due to exponential decay, the integrand decreases very rapidly as $k$ increases. Satisfactory accuracy is achieved by taking the initial six to seven zeros of the integrand. Figure 8 demonstrates the diffusion of the pore pressure in the poroelastic half-space with time for $x=h, z=0, h, 2 h$. The rate of diffusion increases with distance from the interface. Figure $9(\mathrm{a}, \mathrm{b})$ depicts the variation of the pore pressure with the horizontal distance from the fault for $z=0, h$ and $T=0$ (undrained state), 0.1, 1 , 
10 and $\infty$ (drained state). Of course, $P=0$ in the drained state. The point of maximum pore pressure moves away from the source with time. Figure 10 shows the variation of the pore pressure in the poroelastic half-space with distance from the interface for $x=h, G / G^{\prime}=2$ and $T=0$ (undrained state), $0.1,1,10$ and $\infty$ (drained state).

\section{Acknowledgements}

The authors are thankful to the referees for their comments which led to this improved version of the paper. One of the authors (SJS) is grateful to the Indian National Science Academy for financial support through its Senior Scientist Scheme.

\section{Appendix}

\section{Notation}

$B$ Skempton's coefficient

c hydraulic diffusivity

$G$ shear modulus

$m=\left(k^{2}-\iota \omega / c\right)^{1 / 2}$

$p$ pore pressure (compression negative)

$P_{1}=\frac{1-\theta}{1+3 \theta-4 \theta \nu^{\prime}}, \quad P_{2}=P_{1}-1$

$P_{3}=\frac{4 \nu_{u}-3-\theta}{1-\theta}, \quad P_{4}=\frac{4 \nu-3-\theta}{1-\theta}$

$q$ Darcy's flux in the $z$-direction

$Q=\frac{P_{2}}{P_{1}\left(P_{3}+\Omega\right)}, \quad Q_{1}=\frac{P_{2}}{P_{1} P_{4}}, \quad Q_{2}=\frac{P_{2}}{P_{1} P_{3}}$

$Q_{3}=Q_{1}(1-\nu), \quad Q_{4}=Q_{2}\left(1-\nu_{u}\right)$

$R_{1}^{2}=x^{2}+(z+h)^{2}$

$R_{2}^{2}=x^{2}+(z-h)^{2}$

$t$ time

$Z=z+h$

$\alpha \quad$ Biot-Willis coefficient

$$
=\frac{3\left(\nu_{u}-\nu\right)}{B(1-2 \nu)\left(1+\nu_{u}\right)}
$$

$\alpha^{\prime}=\frac{1}{2\left(1-\nu^{\prime}\right)}$

$\alpha_{0}=\frac{3\left(\nu_{u}-\nu\right)}{B\left(1+\nu_{u}\right)}$

$\gamma=\frac{2\left(\nu-\nu_{u}\right)}{1-\nu}$

$\epsilon= \pm 1$ for $Z \gtrless 0$ $\nu \quad$ drained Poisson's ratio

$\nu_{u} \quad$ undrained Poisson's ratio

$\eta=\frac{3\left(\nu_{u}-\nu\right)}{2 B(1-\nu)\left(1+\nu_{u}\right)}=\frac{1-2 \nu}{2(1-\nu)} \alpha$

$\theta=G / G^{\prime}$

$\chi \quad$ Darcy conductivity

$$
=\frac{9 c\left(1-\nu_{u}\right)\left(\nu_{u}-\nu\right)}{2 G B^{2}(1-\nu)\left(1+\nu_{u}\right)^{2}}
$$

$\Omega=\frac{k^{2} \gamma}{m(m+k)}$

\section{References}

Ben-Menahem A and Singh S J 1981 Seismic Waves and Sources (Springer-Verlag, New York) pp. 1108.

Biot M A 1941 General theory of three-dimensional consolidation; J. Appl. Phys. 12 155-164.

Biot M A 1956 General solutions of the equations of elasticity and consolidation for a porous material; J. Appl. Mech. 78 91-98.

Chau K T 1996 Fluid point source and point forces in linear elastic diffusive half-spaces; Mech. Mater. 23 241-253.

Heaton T H and Heaton R E 1989 Static deformation from point forces and point force couples located in welded elastic Poissonian half-spaces: implication for seismic moment tensors; Bull. Seismol. Soc. Am. 79 813-841.

Kumari G, Singh S J and Singh K 1992 Static deformation of two welded elastic half-spaces caused by a point dislocation source; Phys. Earth Planet. Int. 73 53-76.

Maruyama T 1966 On two-dimensional elastic dislocations in an infinite and semi-infinite medium; Bull. Earthq. Res. Inst. 44 811-871.

Pan E 1999 Green's functions in layered poroelastic half-spaces; Int. J. Numer. Anal. Meth. Geomech. 23 1631-1653.

Rajapakse R K N D and Senjuntichai T 1993 Fundamental solutions for a poroelastic half-space with compressible constituents; J. Appl. Mech. 60 844-856.

Rice J R and Clearly M P 1976 Some basic stress diffusion solutions for fluid-saturated elastic porous media with compressible constituents; Rev. Geophys. Space Phys. 14 $227-241$.

Rongved L 1955 Force interior to one of two joined semi-infinite solids; In: Proceedings of the 2nd Midwestern Conference on Solid Mechanics, Purdue University, Indiana (ed.) Bogdanoff J L, Res. Ser. 129 1-13.

Rudnicki J W 1986 Fluid mass sources and point forces in linear elastic diffusive solids; Mech. Mater. 5 383-393.

Rudnicki J W 1987 Plane strain dislocations in linear elastic diffusive solids; J. Appl. Mech. 54 545-552.

Rudnicki J W and Roeloffs E 1990 Plane-strain shear dislocations moving steadily in linear elastic diffusive solids; J. Appl. Mech. 57 32-39.

Schapery R A 1962 Approximate methods of transform inversion for viscoelastic stress analysis; Proc. 4th U.S. Nat. Congress on Appl. Mech. 2 1075-1085.

Senjuntichai T and Rajapakse R K N D 1995 Exact stiffness method for quasi-statics of a multi-layered poroelastic medium; Int. J. Solids Struct. 32 1535-1553. 
Singh S J and Garg N R 1986 On the representation of two-dimensional seismic sources; Acta Geophys. Polon. 34 1-12.

Singh S J and Rani S 2006 Plane strain deformation of a multilayered poroelastic half-space by surface loads; J. Earth Sys. Sci. 115 685-694.
Singh S J, Rani S and Garg N R 1992 Displacements and stresses in two welded half-spaces due to twodimensional sources; Phys. Earth Planet. Inter. 70 90-101.

Wang H F 2000 Theory of Linear Poroelasticity (Princeton Univ. Press, Princeton) pp. 287.

MS received 23 March 2006; revised 28 October 2006; accepted 10 November 2006 